\title{
VEHICLE TO VEHICLE RF PROPAGATION MEASUREMENTS
}

This text presents results from vehicle to vehicle RF propagation measurements in the $900 \mathrm{MHz}$ band focusing on determining delay spread, probability distribution parameters (in particular, the Rician $K$ factor) and path loss rates. The parameters and results are discussed with implications for the use of RF communication between automobiles in Intelligent Vehicle/Highway Systems (IVHS). 


\subsection{Introduction}

The purpose of this text is to present propagation measurements of RF signals between vehicles. The results help determine the feasibility of using RF communication between vehicles in IVHS platoons. The organization of this text is as follows. Section 3.2 is a review of RF propagation theories. Section 3.3 outlines the measurement procedure and presents the measurement results. Section 3.4 discusses the results and presents some conclusions.

\subsection{RF Propagation Review}

A great deal of literature is available on RF mobile propagation [1 - 16]. Much of the work has been focused on transmission between users in automobiles to and from a shared base station $[1,7,8]$. In such scenarios, transmission distances can be quite large, often measured in terms of city blocks. Furthermore, a line of sight (LOS) path between the transmitter and the receiver is not always likely as the base station antenna is often blocked by buildings and other obstacles. The channel is quite different when considering vehicle to vehicle communication. Based on the proposed platoon structures of IVHS designs, it can be assumed that the transmission distances will be quite small compared to the RF transmission cases cited above. In addition, a LOS signal path is virtually guaranteed since any obstacles in the roadway would not only be harmful to RF transmission, but also to driver safety.

There are several causes of signal corruption in a wireless channel. Fundamentally, three of the primary causes of corruption are signal attenuation due to distance, multipath reception and channel time variation. Signal attenuation over distance is observed when the mean received signal power is attenuated as a function of the distance from the transmitter. The most common form of this is often called free space loss and is due to the signal power being spread out over the surface area of an expanding sphere as the receiver moves farther from the transmitter.

Multipath transmission results from the fact that the transmission channel consists of several obstacles and reflectors. Thus, the received signal arrives as a set of reflections and/ or direct waves each with its own degree of attenuation and delay. Delay spread, $T_{D}$, is a parameter commonly used to quantify multipath effects. Informally, delay spread is the difference in time between the first and last 
received signals from the transmitter to the receiver. More formally we consider the RMS and Mean Excess delay spread in which the instantaneous impulse response is treated like a probability distribution function (pdf). Multipath transmission manifests itself as variation in the received signal strength over frequency. Often researchers consider the coherence bandwidth as a measure of how much the received power level varies in the frequency domain. Coherence bandwidth appears to be inversely proportional to the delay spread.

Time variation of the channel is due to the assumption that the communicating vehicles are in motion. Closely related to Doppler shifting, time variation in conjunction with multipath transmission leads to variation of the instantaneous received signal strength about the mean power level as the receiver moves over distances on the order of less than a single carrier wavelength. Given that the vehicles are in motion, time variation of the channel is realized as spatial variation and becomes uncorrelated approximately every half carrier wavelength over distance.

The variation of received signal amplitude in the time domain observes a probability distribution in one of two forms. The first form, Rayleigh fading, generally occurs when there is no direct line-of-sight path between the transmitter and the receiver. The second and more general form of multipath fading is Rician fading and occurs when a line-of-sight path is present. A Rician distribution consists of two parameters; the Rician $K$ factor and local mean signal power. The $K$ factor is defined as the ratio of the direct line-of-sight signal strength to the scattered (reflected) signal components. For Rayleigh fading, the $K$ factor is zero and thus the Rician distribution reduces mathematically to a Rayleigh distribution.

\subsubsection{Base Station to Vehicle Channel Mode I}

The correct reception of transmitted data depends on the signal power relative to the background noise in the channel, and any interfering signals. It is hence important to characterize the spatial power variation in the channel. Four propagation mechanisms influence mobile radio reception:

1. Large Scale Attenuation.

2 The Shadowing Effect. 


\section{Channelling Effects.}

\section{Multipath Fading.}

\subsubsection{Large Scale Attenuation}

We model the propagation channel as a dominant direct component, with an amplitude determined by path loss, a set of early reflected waves adding coherently with the dominant wave, and intersymbol interference caused by the excessively delayed waves, adding incoherently with the dominant wave. We learn from propagation models proposed for micro-cellular communication that the path loss shows a transition from free-space loss to a ground wave propagation. Free-space loss is experienced if $r \lambda<4 h_{r} h_{t}$ where $r$ represents the distance from the transmitter, $\lambda$ is the wavelength of the carrier wave, and $h_{r} \& h_{t}$ are respectively the heights of the receiving and transmitting antennas. Harley [20] suggested a smooth transition, with

$$
\bar{p}=r^{-\beta_{1}}\left(1+\frac{r}{r_{g}}\right)^{-\beta_{2}}
$$

where $r_{g}$ is the normalized turn over distance, and $p$ is the local-mean power (i.e., received power averaged over a few meters). Studies done by [15] indicate that actual turn over distances on the order of 800 meters are reasonable around $2 \mathrm{GHz}$. Other models, such as a stepwise transition, have been proposed. Empirical values for $\beta_{1}$ and $\beta_{2}$ have been reported e.g. [21].

\subsubsection{Shadowing Effect}

The shadowing effect refers to the fluctuation of the local-mean power about the area-mean power (i.e., received power averaged over tens to hundreds of meters) due to large scale reflections. Due to the limited (micro-cellular) transmission distances involved with the Intelligent Vehicle/Highway Systems, there will generally be a line-of-sight (LOS) path between the transmitter and receiver. A factor to consider is obstruction due to traffic on the highway. Studies in [15] show that the shadowing effects in the IVHS environment do not necessarily give rise to the log-normal distribution of the local-mean power as is the case in the usual macro-cellular environments. Further, shadowing and 
multipath appear not to behave as independent processes [15]. Calculations in [15] to determine the received power involve determining which rays are obstructed (if any) and subsequently using a specific Multi-Ray model. In the extreme case of an obstruction of the LOS and ground waves, the power is Rayleigh distributed. In this text, we will make the assumption that there is always a line-of-sight path.

\subsubsection{Channelling Effects}

Specular reflections from near and far walls that are typical along highways lead to fairly quick variations of power with distance [15].

\subsubsection{Multipath Fading}

Road side base stations may be assumed to be transmitting to vehicles within distances on the order of a few hundred meters. The channel is also characterized by the presence of multipath reflections due to the relatively low antenna positions. The received signals hence consist of a dominant line-of-sight component and reflections of the transmitted signals. This leads to rapid power fluctuations as the vehicle moves within the coverage area of the base station. In this text, only narrowband channels are considered. This implies that the interarrival times of the reflected waves are small compared to the variations in the modulating signal. The reflected waves hence add coherently. RMS delay spreads on the order of 1 ns have been computed [15] for the IVHS environment at carrier frequencies around $1800 \mathrm{MHz}$. The coherence bandwidth is hence around $100 \mathrm{MHz}$. Given the assumption of bit rates of up to $80 \mathrm{kHz}$ between vehicles and base stations, the narrowband assumption is reasonable. RMS delay spreads longer than $1 \mathrm{~ns}$ have been reported; e.g., $300 \mathrm{~ns}$ is reported for small city microcells [22]. The narrowband assumption is still valid for this value.

Rician fading occurs in a channel if the central limit theorem can be applied to the reflected waves. This in turn only occurs if the number of reflected waves is large, and none of them dominates the joint reflected power. Considering the characteristics of the channel described above, Rician fad- 
ing is a good assumption.

\subsubsection{Vehicle to Vehicle Channel Mode I}

\subsubsection{Attenuation Models}

Vehicle-to-vehicle data communication will mainly consist of the continuous (routine) exchange of telemetric data such as vehicle status, speed, and acceleration. Interfering signals will be present from vehicles within the platoon and from outside the platoon (from vehicles in other lanes). Vehicles with bumper mounted directional antennas are considered.

The vehicle-to-vehicle radio link can be modelled statistically as a Rician fading channel. The dominant component in the Rician fading channel is likely to be relatively strong compared to the reflected signal (large Rician $K$-factor), and the delay spread is likely to be relatively small because reflections occur in the immediate vicinity of the transmitter and receiver antenna. The propagation channel is modelled as a dominant component consisting of a direct-line-of-sight wave and a ground reflected wave, a set of early reflected waves, and intersymbol interference caused by excessively delayed waves.

Propagation models proposed for micro-cellular communication, model path loss with a transition from free-space propagation to groundwave propagation if $d \lambda<4 h_{r} h_{t}$, where $d$ is the distance of the radio link under study, $h_{r}$ and $h_{t}$ are the heights of the receiving and transmitting antenna respectively, and $\lambda$ is the wavelength of the transmitted wave. Various models have been proposed, e.g. a step-wise transition from $\beta_{1}=2$ to $\beta_{2}=4$ (empirical values) at a certain (turnover) distance $d_{g}$. Harley's [20] smooth transition, has been described earlier. However when the distance between the receiver and antenna is small and unobstructed the direct line-of-sight component and the ground reflected component will cause strong fluctuations in the received signal power due to mutual interference between these two waves. Thus the local mean power of the dominant wave does not show a smooth transition between free-space and groundwave propagation. Rather this transition is marked by strong fluctuations in the local mean power. 


\subsubsection{Path Loss Near a Dielectric Surface}

The road surface is neither a perfect conductor nor dielectric so the reflection coefficient depends on the dielectric constant $\varepsilon$ and the conductivity $\sigma$ of the road surface. In order to facilitate computation we assume the road surface to be smooth and thus the dielectric constant and conductivity do not vary with distance. The reflection coefficient for horizontally polarized waves is given by [23]

$$
\Gamma=\frac{\sin \Theta-\sqrt{\left(\frac{\varepsilon}{\varepsilon_{0}}-\mathrm{j} \frac{\sigma}{\omega \varepsilon_{0}}\right)-(\cos \Theta)^{2}}}{\sin \Theta+\sqrt{\left(\frac{\varepsilon}{\varepsilon_{0}}-\mathrm{j} \frac{\sigma}{\omega \varepsilon_{0}}\right)-(\cos \Theta)^{2}}},
$$

where $\omega$ is the angular frequency of the signal, $\varepsilon_{0}$ is the dielectric constant of free space and $\Theta$ is the angle of incidence, which we assume to be equal to the angle of reflection, and

$$
\chi=\frac{\sigma}{\omega \varepsilon_{0}}=\frac{18 \times 10^{9} \sigma}{f}
$$

For vertical polarization the reflection coefficient is given by [23]

$$
\Gamma=\frac{\left(\varepsilon_{r}-\mathrm{j} \chi\right) \sin \Theta-\sqrt{\left(\varepsilon_{r}-\mathrm{j} \chi\right)-(\cos \Theta)^{2}}}{\left(\varepsilon_{r}-\mathrm{j} \chi\right) \sin \Theta+\sqrt{\left(\varepsilon_{r}-\mathrm{j} \chi\right)-(\cos \Theta)^{2}}}
$$

Since this reflection coefficient is complex, the reflected ground wave will differ in both amplitude and phase. The phase difference of the two paths is [14]

$$
\Delta \varphi=\frac{2 \pi}{\lambda}\left\{\sqrt{d^{2}+\left(h_{t}+h_{r}\right)^{2}}-\sqrt{d^{2}+\left(h_{t}-h_{r}\right)^{2}}\right\} .
$$

If the field strength at the receiving antenna due to the direct line-of-sight wave is $E_{0}$, then the received field due to the sum of the direct line-of-sight component and ground reflected component i s

$$
E=E_{0}[1+|\Gamma| \exp (j \angle \Gamma-j \Delta \varphi)]=E_{0}[1+|\Gamma| \cos (\angle \Gamma-\Delta \varphi)+j|\Gamma| \sin (\angle \Gamma-j \Delta \varphi)]
$$

Taking the absolute value we find that

$$
|E|=\left|E_{0}\right|\left[1+|\Gamma|^{2}+2|\Gamma| \cos (\angle \Gamma-j \Delta \varphi)\right]^{1 / 2}
$$


and since the received power $p_{r}$ is proportional to the square of the received energy we have

$$
p_{r}=\left|E_{0}\right|^{2}\left[1+|\Gamma|^{2}+2|\Gamma| \cos (\angle \Gamma-j \Delta \varphi)\right]
$$

and

$$
\frac{p_{r}}{p_{t}}=\left(\frac{\lambda}{4 \pi d}\right)^{2} G_{t} G_{r}\left[1+|\Gamma|^{2}+2|\Gamma| \cos (\angle \Gamma-j \Delta \varphi)\right] .
$$

If $d \gg h_{t} h_{r}$, i.e. the angle of incidence becomes small, the reflection coefficient $\Gamma \rightarrow-1$. Thus (7) becomes

$$
|E|=2\left|E_{0}\right| \sin \frac{\Delta \varphi}{2}
$$

Then using the small angle approximation $\sin \Delta \varphi \approx \Delta \varphi$ and further expressing $\Delta \varphi \approx\left(4 \pi h_{t} h_{r}\right) /(\lambda d)$, both valid approximations for a large separation distance, (9) can be expressed as

$$
\frac{p_{r}}{p_{t}}=G_{t} G_{r}\left(\frac{4 \pi h_{t} h_{r}}{d^{2}}\right)^{2}
$$

\subsection{Measurement Setup and Results}

The experiments were primarily concerned with measurement of delay spread, Rician $K$ factor and path loss versus distance; under various scenarios of automobile and antenna positions. The measurements were taken under stationary conditions on a roadway environment with parked cars. The antennas were supported by tripods at a height approximating an automobile's front hood. Two automobiles were parked in tandem at particular separation distance. As this distance was varied (from 6 to 40 feet), the parameters of interest were measured. At each distance, six measurements were taken at slightly different positions (by one half of the carrier wavelength to become uncorrelated) to average out fading.

The measurement setup consisted of four components as shown in Fig. 3.1. The components are omnidirectional discone (receive and transmit) antennas, coaxial cables, a microwave network analyzer and a portable PC. The microwave network analyzer served as a signal generator and could 
cover a frequency range of $50 \mathrm{MHz}$ to $13.5 \mathrm{GHz}$ with a maximum frequency resolution of $100 \mathrm{kHz}$. The network analyzer automatically calibrated out the coaxial cable loss. All tests had a transmit power level of $+10 \mathrm{dBm}$. The network analyzer provided frequency response as well as the time domain instantaneous impulse response data corresponding to a given frequency response sweep. This data was ported to the PC for analysis via a GPIB connection.

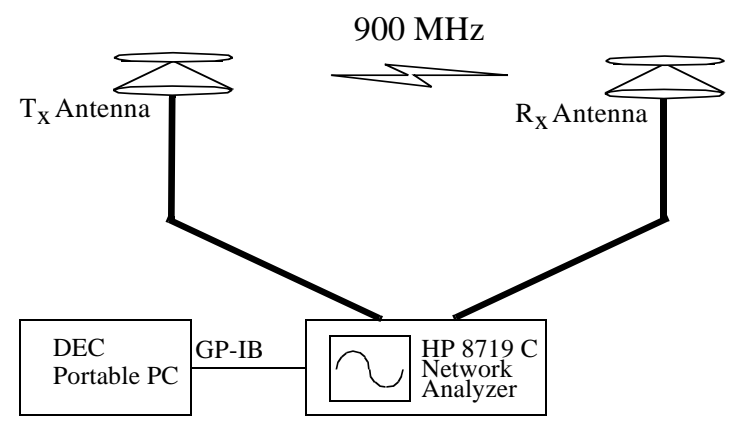

Figure 3.1 Measurement Setup

\subsubsection{Path Loss Attenuatio n}

Path loss rate was anticipated to be very close to, or perhaps slightly smaller than that of free space loss. As Fig. 3.2 shows, the measured rate was 2.36. A possible explanation for this somewhat large path loss value could be due to the inclusion of the measurement taken at the shortest distance. Indeed, with a carrier wavelength on the order of 1 foot in length ( 0.3333 meters to be exact), the short distance of 6 feet separating the two antennas comes somewhat close to the point at which antennas cease to act as "point sources": the antennas are clearly no longer in the far field. If this is the case, the path loss rate no longer follows the simple inverse square law. Indeed, when the path loss was calculated without the first point of Fig. 3.2, the new rate was 1.53. This question of being in the antennas far field was a primary reason for why measurements significantly closer (than 6 feet) were 
not taken.

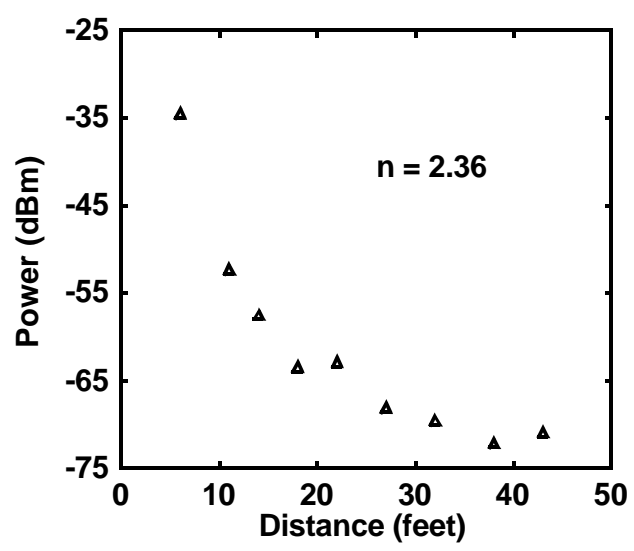

Figure 3.2 Path Loss

\subsubsection{Delay Sprea d}

Delay spread was measured using the technique presented in [15]. There are two interesting issues to note with the delay spread measurements in this experiment. First, the RMS delay spreads are, quite reasonably, very small. Compared to similar indoor wireless measurements that were have taken at $2.4 \mathrm{GHz}$ with the same antenna separation distance, the vehicle to vehicle measurements have significantly smaller delay spreads (as much as a factor of 2). It is inferred that this difference is not due to the difference in carrier frequency but rather a difference in the two types of channel. One case being a relatively tame channel between two closely spaced automobiles, and the other being a relatively cluttered office cubicle environment. Given this difference, it seems quite reasonable that the vehicle to vehicle channel provides few obstacles that can lead to significant reflections, which is the primary cause of delay spreads.

A second interesting point is that the delay spread values increase slightly with distance but in a consistent oscillating pattern. As shown in Fig. 3.3, the RMS delay spread has an oscillating pattern and no four consecutive points can be connected by a monotonic curve. Referring to the concurrent indoor measurements done as part of this experiment as well as those of others $[15,18]$, it should be noted that this is uncharacteristic. Recall that each data point in Fig. 3.3 represents six separate measurements taken within a small region to average out the effects of fading. 
One explanation could be that the relative received signal strength of "long" reflections in the channel oscillates as a function of antenna separation distance. The significant objects in our channel are simply two consecutive vehicles and a flat roadway. Furthermore, the test environment did not have any objects above the vehicles or to the left or right that were close enough to have a serious impact on the channel.

This observation has implications for many of the n-ray models (for integer $\mathrm{n}$, typically small) that are being proposed to characterize channels. Models which describe a wireless channel as having a small, finite number of signal paths can afford an almost deterministic approach due to the reduced number of variables to consider. In effect, it may become possible to "predict" locations at which certain reflections may be cancelled out. It is this kind of prediction that would lend itself to explaining Fig. 3.3.

The oscillations in Fig. 3.3 diminish as distance increases. This makes sense since a large antenna separation distance should overshadow the variations that occur on the order of a wavelength. Additionally, greater distances allow for more reflections which reduces the significance of any single reflection. Another explanation is the effect of a LOS path plus a ground reflection as the dominant components of the Rician channel model. One can view the channel as a phasor sum of: a) LOS, b) strong ground reflection, and c) many random scatters. The RMS delay spread tends to be inversely correlated with the amplitude of the two strong components.

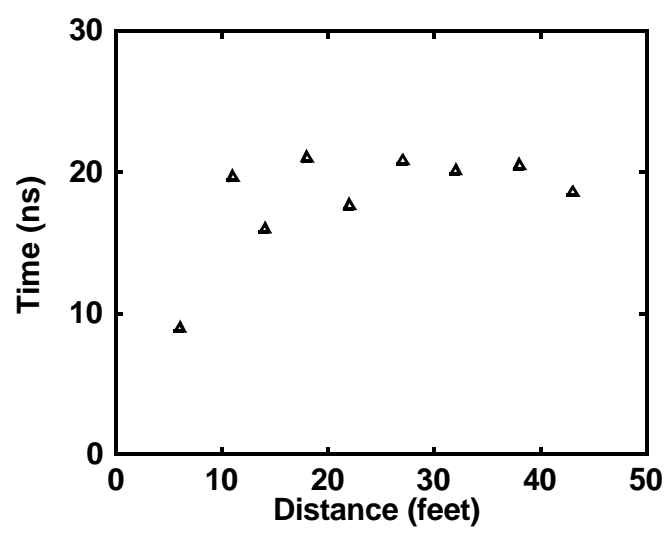

Figure 3.3 RMS Delay Spread vs. Antenna Separation Distance 


\subsubsection{Rician $K$ Factor}

The Rician $K$ factor is very important in completing the knowledge about the associated probability density function of a wireless channel. Indeed, to know the distribution of a wireless channel without knowing the parameters about which the distribution occurs is useless. Knowledge of the Rician $K$ Factor can be useful in determining the bit error rate of a channel among other useful metrics.

Note that the search for a Rician $K$ factor implicitly suggests a Rician distribution has been assumed. Given the intuition that a Rician fading channel has a strong LOS component, this assumption is readily justified.

The method of moments was used to determine the Rician $K$ factor. This method sets the sample mean equal to the theoretical mean (both of the received signal amplitude). The following equation shows the theoretical mean of a Rician distributed random variable.

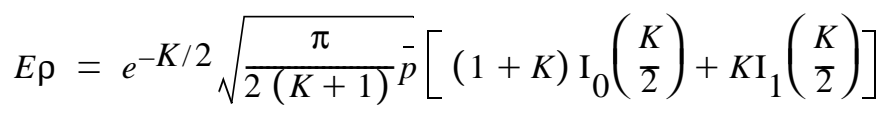

Since both the local mean power, $\bar{p}$, and the sample mean, E $\rho$, are empirically determined, (12) can be used to solve for $K$.

The largest measured $K$ factor was 17.6. This value occurred several times. More typical values were in the range of 5.0 to 11.0. Large $K$ factors were anticipated due to the obvious strong LOS signal that is present between the two vehicles. Contrary to anticipation, however, were occasional measured $K$ factors around 1.5 (the lowest was 1.38).

Recall that the $K$ factor represents the ratio of the received LOS component to the received scattered components. This concept of $K$ factor, first put forth in [13], is very difficult to measure in a physically meaningful manner; i.e., by isolating the direct signal from the scattered components. Since the $K$ factor can serve as a metric for differentiating Rician from Rayleigh distributions, the question becomes, "how large must $K$ be to truly have a Rician distribution?"

It is inferred that the consistency of larger measured $K$ factors (i.e., 11.1, 17.6, etc.) is evidence of a Rician distribution. However, the few low values that were measured does cause concern. It 
seems more likely that the channel is Rician with a dominant component consisting of at least two paths; an LOS and a reflection off of the roadway. These two components may be interacting with one another in such a way as to have a sinusoidal type of frequency response which affects the computations.

As part of future work, alternative method for measuring the $K$ factor can be employed. One, very nonexplicit method for accomplishing this task is to match various (with differing parameters) Rician distribution functions to the tabulated results from the measurements. The distribution (and corresponding $K$ factor) that most closely matches is optimum.

\subsection{Conclusion}

The results of RF wireless vehicle to vehicle measurements at close range are: path loss with an exponential drop off rate of 2.36 and RMS delay spread with a range of values between 8.9 to $20.8 \mathrm{~ns}$. Of note is the RMS delay spreads that had an "oscillating" behavior that can lend itself to many of the n-ray channel characterization models. Additionally, the Rician $K$ factor results mostly range from 5.0 to 11.0 with extreme values as high as 17.6 and as low as 1.38 . The channel is not strictly Rician. Unlike the traditional view of a Rician channel, it is inferred that the channel consists of two dominant components: a LOS signal and a strong reflection off of the roadway. Over distance the two dominant components sum up in a oscillatory manner.

\subsection{References}

[1] R.J.C. Bultitude and G.K. Bedal, "Propagation characteristics on microcellular urban radio channels at 910 MHz", IEEE Jour. Sel. Areas in Commun., vol. 7, no. 1, 1989, pp. 31-39.

[2] D.C. Cox, "Multipath Delay Spread and Path Loss Correlation for 910-MHz Urban Mobile Radio Propagation", IEEE Trans. on Veh. Technol., vol. 26, no. 4, Nov. 1977, pp. 340-344.

[3] D.C. Cox and R.P. Leck, "Correlation bandwidth and delay spread multipath propagation statistics for 910-MHz urban mobile radio channels", IEEE Trans.on Commun., vol. 23, no. 11, Nov. 1975, pp. 1271-80.

[4] D.C. Cox and R.P. Leck, "Distributions of multipath delay spread and average excess delay for 910-MHz urban mobile radio paths", IEEE Trans.on Antennas and Propagation, vol. 23, no.3, 
Mar. 1975, pp. 206-13.

[5] D.M.M. Devasirvatham, "Multipath time delay spread in the digital portable radio environment”, IEEE Commun. Mag., vol. 25, no. 6, June 1987, pp. 13 - 21.

[6] R. Ganesh and K. Pahlavan, "Effects of traffic and local movements on multipath characteristics of an indoor radio channel", Electronics Letters, vol. 26, no. 12, 7th June 1990, pp. 810 812.

[7] H. Hashemi, “The indoor radio propagation channel", Proc. IEEE, vol. 81, no. 7, July 1993, pp. 941 - 968.

[8] S. Howard and K. Pahlavan, "Doppler spread measurements of the indoor radio channel", Electronics Letters, vol. 26, no. 2, 1990, pp. 107-109.

[9] W.C. Jakes (Ed.), Microwave Mobile Communications, New York: Wiley, 1974.

[10] W.C.Y. Lee, Mobile Communications Design Fundamentals, Indianapolis: Sams, 1986.

[11] J.P.M.G. Linnartz, Narrowband Land-Mobile Radio Networks, Boston: Artech House, 1993.

[12] D. Molkdar, "Review on radio propagation into and within buildings", IEE Proceedings-H, vol. 138, no. 1, Feb. 1991, pp. 61-73.

[13] K.A. Norton, L.E. Vogler, W.V. Mansfieldand P.J. Short, "The probability distribution of the amplitude of a constant vector plus a Rayleigh-distributed vector", Proc. of the IRE, Oct. 1955, pp. 1354-1361.

[14] J.D. Parsons, The Mobile Radio Propagation Channel, New York: Wiley, 1992.

[15] A. Polydoros, et al., Vehicle to Roadside Communications Study, PATH Research Report PRR93-4, June 1993.

[16] J.G. Proakis, Digital Communications, 2nd ed., New York: McGraw-Hill, 1989.

[17] T.S. Rappaport, "Characterization of UHF multipath radio channels in factory buildings", IEEE Trans. on Antennas and Propagation, vol. 37, no. 8, Aug. 1989, pp. 1058 - 1069.

[18] A.M. Saleh and R.A. Valenzuela, "A statistical model for indoor multipath propagation”, IEEE J. Sel. Areas in Commun., vol. 5, no. 2, Feb. 1987, pp. 128-137.

[19] W.-C. Wong, "Packet reservation multiple access in a metropolitan microcellular radio environment”, IEEE J. Sel. Areas in Commun., vol. 11, no. 6, Aug. 1993.

[20] H. Harley, "Short distance attenuation measurements at $900 \mathrm{MHz}$ and $1.8 \mathrm{GHz}$ using low antenna heights for microcells," IEEE J. Sel. Areas in Commun., vol. 7, no. 1, 1989, pp. 5-10.

[21] J.P.M.G. Linnartz, "The modelling of diffraction, reflection, and clutter loss in UHF radio propagation", FEL-TNO report 1988-28, The Hague, The Netherlands, 1988.

[22] D.M.J. Devasirvatham, "Radio propagation studies in a small city for universal portable communications", 38th IEEE Vehicular Technology Conf., Philadelphia, PA, June 15-17, 1988, pp. 100-104.

[23] E.C. Jordan and K.G. Balmain, Electromagnetic Waves and Radiating Systems, New York: Prentice-Hall, 1968. 